\title{
On the existence of a mild solution for impulsive hybrid fractional differential equations
}

Huanmin Ge and Jie Xin*

\section{"Correspondence:}

fdxinjie@sina.com

School of Mathematics and

Statistics Science, Ludong

University, Yantai, 264025, China

\begin{abstract}
This paper is motivated by some recent contributions on the existence of solution of impulsive fractional differential equations and the theory of fractional hybrid differential equations by Agarwal, Ahmad, Baleanu, Benchohra, Fečkan, Nieto, Sun, Bai, Zhou, Zhang and Wang. Here, we derive new existence results of a mild solution of impulsive hybrid fractional differential equations. Finally, an example is given to illustrate the result.
\end{abstract}

Keywords: Caputo fractional derivative; impulsive hybrid differential equations; existence

\section{Introduction}

The study of differential equations of fractional order is motivated by the intensive development of the theory of fractional calculus itself (see [1-7]) and the application of fractional differential equations in the modeling of many physical phenomena. There have been many works on the theory of fractional calculus and applications of it. Fractional differential equations, including Riemann-Liouville fractional derivative or Caputo fractional derivative, have received more and more attention (see [8-18]).

In recent years, hybrid differential equations have attracted much attention. The theory of hybrid differential equations has been developed, and we refer the readers to the articles [19-22]. The authors [23] discussed the following fractional hybrid differential equations involving Riemann-Liouville differential operators:

$$
\left\{\begin{array}{l}
{ }^{L} D_{0, t}^{q}\left[\frac{u(t)}{f(t, u(t))}\right]=g(t, u(t)), \quad \text { a.e. } t \in J:=[0, T] \\
u(0)=0,
\end{array}\right.
$$

where ${ }^{L} D_{0, t}^{q}$ is the Riemann-Liouville fractional derivative of order $q \in(0,1)$ with the lower limit zero, $f \in C(J \times \mathbb{R}, \mathbb{R} \backslash\{0\})$ and $g \in C(J \times \mathbb{R}, \mathbb{R})$. They developed existence of solutions under mixed Lipschitz and Carathéodory conditions. Moreover, they have established some fundamental fractional differential inequalities and the comparison principle. Some recent papers have treated the problem of the existence of solutions for impulsive fractional differential equations.

○2014 Ge and Xin; licensee Springer. This is an Open Access article distributed under the terms of the Creative Commons Attribution License (http://creativecommons.org/licenses/by/2.0), which permits unrestricted use, distribution, and reproduction in any medium, provided the original work is properly cited. 
The authors [24] considered the following basic impulsive Cauchy problems:

$$
\left\{\begin{array}{l}
{ }^{c} D_{0, t}^{q} u(t)=f(t, u(t)), \quad t \in J^{\prime}:=J \backslash\left\{t_{1}, \ldots, t_{m}\right\} \\
\Delta u\left(t_{k}\right)=I_{k}\left(u\left(t_{k}^{-}\right)\right), \quad k=1,2, \ldots, m, \\
u(0)=u_{0}
\end{array}\right.
$$

where ${ }^{c} D_{0, t}^{q}$ is the generalized Caputo fractional derivative of order $q \in(0,1)$ with the lower limit zero and $I_{k}: \mathbb{R} \rightarrow \mathbb{R}$ and $t_{k}$ satisfy $0=t_{0}<t_{1}<\cdots<t_{m}<t_{m+1}=T, u\left(t_{k}^{+}\right)=$ $\lim _{\varepsilon \rightarrow 0^{+}} u\left(t_{k}+\varepsilon\right)$ and $u\left(t_{k}^{-}\right)=\lim _{\varepsilon \rightarrow 0^{-}} u\left(t_{k}+\varepsilon\right)$ represent the right and left limits of $u(t)$ at $t=t_{k}$. They established some sufficient conditions for the existence of solutions.

In the recent paper [25], Herzallah obtained the existence of a mild solution for the fractional order hybrid differential equations:

$$
\left\{\begin{array}{l}
{ }^{c} D_{0, t}^{q}\left[\frac{u(t)}{f(t, u(t))}\right]=g(t, u(t)), \quad \text { a.e. } t \in J \\
u(0)=u_{0} \in \mathbb{R} .
\end{array}\right.
$$

In the present paper, we study the following impulsive hybrid fractional differential equations (IHFDE):

$$
\begin{cases}{ }^{c} D_{0, t}^{q}\left[\frac{u(t)}{f(t, u(t))}\right]=g(t, u(t)), & t \in J^{\prime}:=J \backslash\left\{t_{1}, \ldots, t_{m}\right\}, \\ u\left(t_{k}^{+}\right)=u\left(t_{k}^{-}\right)+I_{k}\left(u\left(t_{k}^{-}\right)\right), & k=1,2, \ldots, m, \\ u(0)=u_{0}, & \end{cases}
$$

where ${ }^{c} D_{0, t}^{q}$ is the generalized Caputo fractional derivative of order $q \in(0,1)$ with the lower limit zero, $f \in C(J \times \mathbb{R}, \mathbb{R} \backslash\{0\})$ and $g \in C(J \times \mathbb{R}, \mathbb{R})$.

This paper is arranged as follows. In Section 2, we recall some concepts and some fractional calculation law and establish preparation results. In Section 3, we give the main results based on the Dhage fixed point theorem. In Section 4, we give an example to demonstrate the application of our main result.

\section{Preliminaries}

In this section, we recall some basic definitions and properties of the fractional calculus theory and preparation results. Throughout this paper, $J_{1}$ denotes the interval $\left[t_{0}, t_{1}\right]$ and $J_{k+1}$ denotes the interval $\left(t_{k}, t_{k+1}\right], k=1,2, \ldots, m$. Let $C(J, \mathbb{R})$ be the Banach algebra of all continuous functions from $J$ into $\mathbb{R}$ with the norm $\|u\|_{c}=\sup \{|u(t)|: t \in J\}$ for $u \in C(J, \mathbb{R})$ and with multiplication $(u v)(s)=u(s) v(s)$ for $u, v \in C(J, \mathbb{R})$. Define $P C(J, \mathbb{R})=\{u: J \rightarrow \mathbb{R}:$ $u \in C\left(\left(t_{k}, t_{k+1}\right], \mathbb{R}\right), k=0,1, \ldots, m$ and there exist $u\left(t_{k}^{-}\right)$and $u\left(t_{k}^{+}\right), k=1,2, \ldots, m$, with $u\left(t_{k}^{-}\right)=$ $\left.u\left(t_{k}\right)\right\}$ with the norm $\|u\|_{P C}:=\sup \{\|u(t)\|: t \in J\}$ that is a Banach space.

We introduce the following known definitions. For more details, one can see [2].

Definition 2.1 The fractional integral of order $\gamma$ with the lower limit zero for a function $f:[0, \infty) \rightarrow \mathbb{R}$ is defined as $I_{t}^{\gamma} f(t)=\frac{1}{\Gamma(\gamma)} \int_{0}^{t} \frac{f(s)}{(t-s)^{1-\gamma}} d x, t>0, \gamma>0$ provided the right-hand side is point-wise defined on $[0, \infty)$, where $\Gamma(\cdot)$ is the gamma function.

Definition 2.2 The Riemann-Liouville derivative of order $\gamma$ with the lower limit zero for a function $f:[0, \infty) \rightarrow \mathbb{R}$ can be written as ${ }^{L} D_{0, t}^{\gamma} f(t)=\frac{1}{\Gamma(n-\gamma)} \frac{d^{n}}{d t^{n}} \int_{0}^{t} \frac{f(s)}{(t-s)^{\gamma+1-n}} d s, t>0$, $n-1<\gamma<n$. 
Definition 2.3 The Caputo derivative of order $\gamma$ for a function $f:[0, \infty) \rightarrow \mathbb{R}$ can be written as

$$
{ }^{c} D_{0, t}^{\gamma} f(t)={ }^{L} D_{0, t}^{\gamma}\left[f(t)-\sum_{k=0}^{n-1} \frac{t^{k}}{k !} f^{(k)}(0)\right], \quad t>0, n-1<\gamma<n .
$$

Remark (i) We have to explain that we follow the ideas from the recent contributions on impulsive fractional differential equations by Fečkan et al. [26, 27].

(ii) In Definition 2.3, the integrable function $f$ can be discontinuous.

(iii) For more details and explanations on such interesting problems, one can refer to [26, Discussions I-V, p.4214] and [27, Remark 2.21(iii)].

Lemma 2.4 ([28]) Let $S$ be a non-empty, closed convex and bounded subset of the Banach algebra $X$, and let $A: X \rightarrow X$ and $B: S \rightarrow X$ be two operators such that

(a) $A$ is Lipschitzian with a Lipschitz constant $\alpha$;

(b) $B$ is completely continuous;

(c) $u_{1}=A u_{1} B u_{2} \Rightarrow u_{1} \in S$ for all $u_{2} \in S$;

(d) $\alpha M<1$, where $M=\|B(S)\|=\sup \{\|B(u)\|: u \in S\}$.

Then the operator equation $A u B u=u$ has a solution in $S$.

Lemma 2.5 ([24]) Let $q \in(0,1)$ and $h:\left[0, T_{0}\right] \mapsto \mathbb{R}$ be continuous. A function $u \in$ $C\left(\left[0, T_{0}\right], \mathbb{R}\right)$ is a solution of the fractional integral equation

$$
u(t)=u_{0}-\frac{1}{\Gamma(q)} \int_{0}^{a}(a-s)^{q-1} h(s) d s+\frac{1}{\Gamma(q)} \int_{0}^{t}(t-s)^{q-1} h(s) d s
$$

if and only if $u$ is a solution of the following fractional Cauchy problems:

$$
\left\{\begin{array}{l}
{ }^{c} D_{0, t}^{q} u(t)=h(t), \quad t \in\left[0, T_{0}\right] \\
u(a)=u_{0}, \quad a>0 .
\end{array}\right.
$$

We introduce the following hypotheses in what follows.

(H1) The function $u \mapsto \frac{u}{f(t, u)}$ is increasing in $\mathbb{R}$ for every $t \in J_{1}$.

(H2) There exists a constant $L_{k}>0$ such that $\left|f\left(t, u_{1}\right)-f\left(t, u_{2}\right)\right| \leq L_{k}\left|u_{1}-u_{2}\right|$ for all $t \in J_{k}(k=1,2, \ldots, m+1)$ and $u_{1}, u_{2} \in \mathbb{R}$.

(H3) There exists a function $h_{k} \in L^{\infty}\left(J_{k}\right)(k=1,2, \ldots, m+1)$ such that $|g(t, u(t))| \leq h_{k}(t), t \in J_{k}(k=1,2, \ldots, m+1)$ for all $u(t) \in \mathbb{R}$.

(H4) $f(t, u(t))$ is continuous on $J_{k}(k=1,2, \ldots, m+1)$ for every $u \in P C(J, \mathbb{R})$.

Lemma 2.6 Assume that hypotheses (H1) and (H4) hold. Let $q \in(0,1)$ and $h: J \rightarrow \mathbb{R}$ be continuous. A function $u$ is a solution of the fractional integral equation

$$
u(t)= \begin{cases}f(t, u(t))\left[\frac{u_{0}}{f\left(0, u_{0}\right)}+\frac{1}{\Gamma(q)} \int_{0}^{t}(t-s)^{q-1} h(s) d s\right], & t \in J_{1}, \\ f(t, u(t))\left[\frac{u_{0}}{f\left(0, u_{0}\right)}+\frac{I_{1}\left(u\left(t_{1}^{-}\right)\right)}{f\left(t_{1}, u\left(t_{1}\right)\right)}+\frac{1}{\Gamma(q)} \int_{0}^{t}(t-s)^{q-1} h(s) d s\right], & t \in J_{2}, \\ f(t, u(t))\left[\frac{u_{0}}{f\left(0, u_{0}\right)}+\frac{I_{1}\left(u\left(t_{1}^{-}\right)\right)}{f\left(t_{1}, u\left(t_{1}\right)\right)}+\frac{I_{2}\left(u\left(t_{2}^{-}\right)\right)}{f\left(t_{2}, u\left(t_{2}\right)\right)}\right. & \\ \left.\quad+\frac{1}{\Gamma(q)} \int_{0}^{t}(t-s)^{q-1} h(s) d s\right], & t \in J_{3}, \\ \vdots & \\ f(t, u(t))\left[\frac{u_{0}}{f\left(0, u_{0}\right)}+\sum_{i=1}^{m} \frac{I_{i}\left(u\left(t_{i}^{-}\right)\right)}{f\left(t_{i}, u\left(t_{i}\right)\right)}+\frac{1}{\Gamma(q)} \int_{0}^{t}(t-s)^{q-1} h(s) d s\right], & t \in J_{m+1},\end{cases}
$$


if and only if $u$ is a solution of the following impulsive problem:

$$
\left\{\begin{array}{l}
{ }^{c} D_{0, t}^{q}\left[\frac{u(t)}{f(t, u(t))}\right]=h(t), \quad t \in J^{\prime}, \\
u\left(t_{k}^{+}\right)=u\left(t_{k}^{-}\right)+I_{k}\left(u\left(t_{k}^{-}\right)\right), \quad k=1,2, \ldots, m, \\
u(0)=u_{0} .
\end{array}\right.
$$

Proof Assume that $u$ satisfies (6). If $t \in J_{1}$, then

$$
\begin{aligned}
& { }^{c} D_{0, t}^{q}\left[\frac{u(t)}{f(t, u(t))}\right]=h(t), \quad t \in J_{1}, \\
& u(0)=u_{0} .
\end{aligned}
$$

Operating by $I_{0, t}^{q}$ on both sides of (7), one can obtain

$$
\begin{aligned}
\frac{u(t)}{f(t, u(t))} & =\frac{u(0)}{f(0, u(0))}+\frac{1}{\Gamma(q)} \int_{0}^{t}(t-s)^{q-1} h(s) d s \\
& =\frac{u_{0}}{f\left(0, u_{0}\right)}+\frac{1}{\Gamma(q)} \int_{0}^{t}(t-s)^{q-1} h(s) d s,
\end{aligned}
$$

i.e.,

$$
u(t)=f(t, u(t))\left[\frac{u_{0}}{f\left(0, u_{0}\right)}+\frac{1}{\Gamma(q)} \int_{0}^{t}(t-s)^{q-1} h(s) d s\right] .
$$

If $t \in J_{2}$, then

$$
\begin{aligned}
& { }^{c} D_{0, t}^{q}\left[\frac{u(t)}{f(t, u(t))}\right]=h(t), \quad t \in J_{2}, \\
& u\left(t_{1}^{+}\right)=u\left(t_{1}^{-}\right)+I_{1}\left(u\left(t_{1}^{-}\right)\right) .
\end{aligned}
$$

According to Lemma 2.5 and the continuity of $f(t, u(t))$, we have

$$
\begin{aligned}
\frac{u(t)}{f(t, u(t))} & =\frac{u\left(t_{1}^{+}\right)}{f\left(t_{1}, u\left(t_{1}\right)\right)}-\frac{1}{\Gamma(q)} \int_{0}^{t_{1}}\left(t_{1}-s\right)^{q-1} h(s) d s+\frac{1}{\Gamma(q)} \int_{0}^{t}(t-s)^{q-1} h(s) d s \\
& =\frac{\left[u\left(t_{1}^{-}\right)+I_{1}\left(u\left(t_{1}^{-}\right)\right)\right]}{f\left(t_{1}, u\left(t_{1}\right)\right)}-\frac{1}{\Gamma(q)} \int_{0}^{t_{1}}\left(t_{1}-s\right)^{q-1} h(s) d s+\frac{1}{\Gamma(q)} \int_{0}^{t}(t-s)^{q-1} h(s) d s .
\end{aligned}
$$

Since

$$
u\left(t_{1}^{-}\right)=f\left(t_{1}, u\left(t_{1}\right)\right)\left[\frac{u_{0}}{f\left(0, u_{0}\right)}+\frac{1}{\Gamma(q)} \int_{0}^{t_{1}}\left(t_{1}-s\right)^{q-1} h(s) d s\right]
$$

there exists

$$
\begin{aligned}
\frac{u(t)}{f(t, u(t))}= & {\left[\frac{u_{0}}{f\left(0, u_{0}\right)}+\frac{1}{\Gamma(q)} \int_{0}^{t_{1}}\left(t_{1}-s\right)^{q-1} h(s) d s\right]+\frac{I_{1}\left(u\left(t_{1}^{-}\right)\right)}{f\left(t_{1}, u\left(t_{1}\right)\right)} } \\
& -\frac{1}{\Gamma(q)} \int_{0}^{t_{1}}\left(t_{1}-s\right)^{q-1} h(s) d s+\frac{1}{\Gamma(q)} \int_{0}^{t}(t-s)^{q-1} h(s) d s \\
= & \frac{u_{0}}{f\left(0, u_{0}\right)}+\frac{I_{1}\left(u\left(t_{1}^{-}\right)\right)}{f\left(t_{1}, u\left(t_{1}\right)\right)}+\frac{1}{\Gamma(q)} \int_{0}^{t}(t-s)^{q-1} h(s) d s,
\end{aligned}
$$


i.e.,

$$
u(t)=f(t, u(t))\left[\frac{u_{0}}{f\left(0, u_{0}\right)}+\frac{I_{1}\left(u\left(t_{1}^{-}\right)\right)}{f\left(t, u\left(t_{1}\right)\right)}+\frac{1}{\Gamma(q)} \int_{0}^{t}(t-s)^{q-1} h(s) d s\right] .
$$

If $t \in J_{3}$, we have

$$
\begin{aligned}
\frac{u(t)}{f(t, u(t))} & =\frac{u\left(t_{2}^{+}\right)}{f\left(t_{2}, u\left(t_{2}\right)\right)}-\frac{1}{\Gamma(q)} \int_{0}^{t_{2}}\left(t_{2}-s\right)^{q-1} h(s) d s+\frac{1}{\Gamma(q)} \int_{0}^{t}(t-s)^{q-1} h(s) d s \\
& =\frac{u\left(t_{2}^{-}\right)+I_{2}\left(u\left(t_{2}^{-}\right)\right)}{f\left(t_{2}, u\left(t_{2}\right)\right)}-\frac{1}{\Gamma(q)} \int_{0}^{t_{2}}\left(t_{2}-s\right)^{q-1} h(s) d s+\frac{1}{\Gamma(q)} \int_{0}^{t}(t-s)^{q-1} h(s) d s .
\end{aligned}
$$

For

$$
u\left(t_{2}^{-}\right)=f\left(t_{2}, u\left(t_{2}\right)\right)\left[\frac{u_{0}}{f\left(0, u_{0}\right)}+\frac{I_{1}\left(u\left(t_{1}^{-}\right)\right)}{f\left(t_{1}, u\left(t_{1}\right)\right)}+\frac{1}{\Gamma(q)} \int_{0}^{t_{2}}\left(t_{2}-s\right)^{q-1} h(s) d s\right],
$$

we have that

$$
\begin{aligned}
\frac{u(t)}{f(t, u(t))}= & \frac{u_{0}}{f\left(0, u_{0}\right)}+\frac{I_{1}\left(u\left(t_{1}^{-}\right)\right)}{f\left(t_{1}, u\left(t_{1}\right)\right)}+\frac{1}{\Gamma(q)} \int_{0}^{t_{2}}\left(t_{2}-s\right)^{q-1} h(s) d s \\
& +\frac{I_{2}\left(u\left(t_{2}^{-}\right)\right)}{f\left(t_{2}, u\left(t_{2}\right)\right)}-\frac{1}{\Gamma(q)} \int_{0}^{t_{2}}\left(t_{2}-s\right)^{q-1} h(s) d s+\frac{1}{\Gamma(q)} \int_{0}^{t}(t-s)^{q-1} h(s) d s \\
= & \frac{u_{0}}{f\left(0, u_{0}\right)}+\frac{I_{1}\left(u\left(t_{1}^{-}\right)\right)}{f\left(t_{1}, u\left(t_{1}\right)\right)}+\frac{I_{2}\left(u\left(t_{2}^{-}\right)\right)}{f\left(t_{2}, u\left(t_{2}\right)\right)}+\frac{1}{\Gamma(q)} \int_{0}^{t}(t-s)^{q-1} h(s) d s,
\end{aligned}
$$

i.e.,

$$
u(t)=f(t, u(t))\left[\frac{u_{0}}{f\left(0, u_{0}\right)}+\sum_{i=1}^{2} \frac{I_{i}\left(u\left(t_{i}^{-}\right)\right)}{f\left(t_{i}, u\left(t_{i}\right)\right)}+\frac{I_{2}\left(u\left(t_{2}^{-}\right)\right)}{f\left(t_{2}, u\left(t_{2}\right)\right)}+\frac{1}{\Gamma(q)} \int_{0}^{t}(t-s)^{q-1} h(s) d s\right] .
$$

If $t \in J_{k+1}(k=3,4, \ldots, m)$, using the same method, we get

$$
u(t)=f(t, u(t))\left[\frac{u_{0}}{f\left(0, u_{0}\right)}+\sum_{i=1}^{k} \frac{I_{i}\left(u\left(t_{i}^{-}\right)\right)}{f\left(t_{i}, u\left(t_{i}\right)\right)}+\frac{1}{\Gamma(q)} \int_{0}^{t}(t-s)^{q-1} h(s) d s\right] .
$$

Conversely, assume that $u$ satisfies (5). If $t \in J_{1}$, then we have

$$
u(t)=f(t, u(t))\left[\frac{u_{0}}{f\left(0, u_{0}\right)}+\frac{1}{\Gamma(q)} \int_{0}^{t}(t-s)^{q-1} h(s) d x\right] .
$$

Then, dividing by $f(t, u(t))$ and applying ${ }^{c} D_{0, t}^{q}$ on both sides of (12), (7) is satisfied. Again, substituting $t=0$ in (12), we have $\frac{u(0)}{f(0, u(0))}=\frac{u_{0}}{f\left(0, u_{0}\right)}$. Since $u \mapsto \frac{u}{f(t, u)}$ is increasing in $\mathbb{R}$ for $t \in J_{1}$, the map $u \mapsto \frac{u}{f(0, u)}$ is injective in $\mathbb{R}$. Then we get (8).

If $t \in J_{2}$, then we have

$$
u(t)=f(t, u(t))\left[\frac{u_{0}}{f\left(0, u_{0}\right)}+\frac{I_{1}\left(u\left(t_{1}^{-}\right)\right)}{f\left(t_{1}, u\left(t_{1}\right)\right)}+\frac{1}{\Gamma(q)} \int_{0}^{t}(t-s)^{q-1} h(s) d x\right]
$$


Then, dividing by $f(t, u(t))$ and applying ${ }^{c} D_{t}^{q}$ on both sides of (13), (9) is satisfied. Again, by (H4), substituting $t=t_{1}$ in (12) and taking the limit of (13), then (13) minus (12) gives (10). If $t \in J_{k+1}(k=2,4, \ldots, m)$, similarly we get

$$
\left\{\begin{array}{l}
{ }^{c} D_{0, t}^{q}\left[\frac{u(t)}{f(t, u(t))}\right]=h(t), \quad t \in\left(t_{k}, t_{k+1}\right] \\
u\left(t_{k}^{+}\right)=u\left(t_{k}^{-}\right)+I_{k}\left(u\left(t_{k}^{-}\right)\right)
\end{array}\right.
$$

This completes the proof.

Now we give the following definition.

Definition 2.7 If a function $u \in P C(J, \mathbb{R})$ satisfies the fractional integral equation

$$
u(t)= \begin{cases}f(t, u(t))\left[\frac{u_{0}}{f\left(0, u_{0}\right)}+\frac{1}{\Gamma(q)} \int_{0}^{t}(t-s)^{q-1} g(s, u(s)) d s\right], & t \in J_{1}, \\ f(t, u(t))\left[\frac{u_{0}}{f\left(0, u_{0}\right)}+\frac{I_{1}\left(u\left(t_{1}^{-}\right)\right)}{f\left(t_{1}, u\left(t_{1}\right)\right)}+\frac{1}{\Gamma(q)} \int_{0}^{t}(t-s)^{q-1} g(s, u(s)) d s\right], & t \in J_{2}, \\ f(t, u(t))\left[\frac{u_{0}}{f\left(0, u_{0}\right)}+\frac{I_{1}\left(u\left(t_{1}^{-}\right)\right)}{f\left(t_{1}, u\left(t_{1}\right)\right)}+\frac{I_{2}\left(u\left(t_{2}^{-}\right)\right)}{f\left(t_{2}, u\left(t_{2}\right)\right)}\right. & t \in J_{3}, \\ \left.\quad+\frac{1}{\Gamma(q)} \int_{0}^{t}(t-s)^{q-1} g(s, u(s)) d s\right], & \\ \vdots & \left.t \in J_{m+1}, u(t)\right)\left[\frac{u_{0}}{f\left(0, u_{0}\right)}+\sum_{i=1}^{m} \frac{I_{i}\left(u\left(t_{i}^{-}\right)\right)}{f\left(t_{i}, u\left(t_{i}\right)\right)}\right.\end{cases}
$$

it is said to be a mild solution of (4).

\section{Main results}

In this section, we prove the existence of a mild solution for IHFDE (4) by Lemma 2.4 and Lemma 2.6.

Theorem 3.1 Assume that hypotheses (H1)-(H4) hold. Further, if

$$
\begin{aligned}
& L_{k}\left[\left|\frac{u_{0}}{f\left(0, u_{0}\right)}+\sum_{i=1}^{k-1} \frac{I_{i}\left(u_{i}\left(t_{i}^{-}\right)\right)}{f\left(t_{i}, u_{i}\left(t_{i}\right)\right)}\right|+\frac{1}{\Gamma(q+1)} \sum_{j=0}^{k}\left(t_{k}-t_{j}\right)^{q}\left\|h_{j+1}\right\|_{L^{\infty}}\right]<1, \\
& \quad k=1,2, \ldots, m, m+1
\end{aligned}
$$

for all $u_{i}(t) \in C\left(J_{i}, \mathbb{R}\right)$ and $u_{i}\left(t_{i}\right)$ is bounded, then IHFDE (4) has a mild solution defined on $J$.

Proof By Lemma 2.6, IHFDE (4) is equivalent to the fractional integral equation (15).

If $t \in J_{1}$, we have

$$
u(t)=f(t, u(t))\left[\frac{u_{0}}{f\left(0, u_{0}\right)}+\frac{1}{\Gamma(q)} \int_{0}^{t}(t-s)^{q-1} g(s, u(s)) d s\right]
$$

Set $X_{1}=C\left(J_{1}, \mathbb{R}\right)$ and

$$
S_{1}=\left\{u \in X_{1} \mid\|u\| \leq N_{1}\right\}
$$


where

$$
N_{1}=\frac{F_{0}\left(\left|u_{0}\right| \Gamma(q+1)+t_{1}^{q}\left|f\left(0, t_{0}\right)\right|\left\|h_{1}\right\|_{L^{\infty}}\right)}{\Gamma(q+1)\left|f\left(0, u_{0}\right)\right|-L_{1}\left(\left|u_{0}\right| \Gamma(q+1)+t_{1}^{q}\left|f\left(0, t_{0}\right)\right|\left\|h_{1}\right\|_{L^{\infty}}\right)},
$$

and $F_{0}=\sup _{t \in J}|f(t, 0)|$.

Define two operators $A_{1}: X_{1} \mapsto X_{1}$ and $B_{1}: S_{1} \mapsto X_{1}$ by

$$
A_{1} u(t)=f(t, u(t)), \quad t \in J_{1}
$$

and

$$
B_{1} u(t)=\frac{u_{0}}{f\left(0, u_{0}\right)}+\frac{1}{\Gamma(q)} \int_{0}^{t}(t-s)^{q-1} g(s, u(s)) d s, \quad t \in J_{1} .
$$

We will show that the operators $A_{1}$ and $B_{1}$ satisfy all the conditions of Lemma 2.4.

First, we will show that $A_{1}$ is a Lipschitz operator on $X_{1}$ with the Lipschitz constant $L_{1}$. Set $u_{1}, u_{2} \in X_{1}$. Then, by hypotheses (H2) and (H3), we have

$$
\begin{aligned}
\left|A_{1} u_{1}(t)-A_{1} u_{2}(t)\right| & =\left|f\left(t, u_{1}(t)\right)-f\left(t, u_{2}(t)\right)\right| \\
& \leq L_{1}\left|u_{1}(t)-u_{2}(t)\right| \leq L_{1}\left\|u_{1}-u_{2}\right\|
\end{aligned}
$$

for all $t \in J_{1}$. Taking supremum over $t$, we have

$$
\left\|A_{1} u_{1}-A_{1} u_{2}\right\| \leq L_{1}\left\|u_{1}-u_{2}\right\|
$$

for all $u_{1}, u_{2} \in X_{1}$.

Next, we will show that $B_{1}$ is a compact and continuous operator on $S_{1}$ into $X_{1}$. We show that $B_{1}$ is continuous on $S_{1}$. Let $\left\{u_{n}\right\} \subseteq S_{1}$ converge to a point $u \in S_{1}$. Then, by the Lebesgue dominated convergence theorem, we have

$$
\begin{aligned}
\lim _{n \rightarrow \infty} B_{1} u_{n}(t) & =\frac{u_{0}}{f\left(0, u_{0}\right)}+\lim _{n \rightarrow \infty} \frac{1}{\Gamma(q)} \int_{0}^{t}(t-s)^{q-1} g\left(s, u_{n}(s)\right) d s \\
& =\frac{u_{0}}{f\left(0, u_{0}\right)}+\frac{1}{\Gamma(q)} \int_{0}^{t}(t-s)^{q-1} \lim _{n \rightarrow \infty} g\left(s, u_{n}(s)\right) d s \\
& =\frac{u_{0}}{f\left(0, u_{0}\right)}+\frac{1}{\Gamma(q)} \int_{0}^{t}(t-s)^{q-1} g(s, u(s)) d s \\
& =B_{1} u(t)
\end{aligned}
$$

for all $t \in J_{1}$. So, we have obtained that $B_{1}$ is continuous on $S_{1}$.

Next we will show that $B_{1}$ is a compact operator on $S_{1}$. We show that $B_{1}\left(S_{1}\right)$ is a uniformly bounded and equicontinuous set in $S_{1}$. Let $u \in S_{1}$ be arbitrary. Then, by hypothesis (H3), we have

$$
\begin{aligned}
\left|B_{1} u(t)\right| & \leq\left|\frac{u_{0}}{f\left(0, u_{0}\right)}\right|+\frac{1}{\Gamma(q)} \int_{0}^{t}(t-s)^{q-1}|g(s, u(s))| d s \\
& \leq\left|\frac{u_{0}}{f\left(0, u_{0}\right)}\right|+\frac{1}{\Gamma(q)}\left(\int_{0}^{t}(t-s)^{q-1} h_{1}(s) d s\right) \\
& \leq\left|\frac{u_{0}}{f\left(0, u_{0}\right)}\right|+\frac{t_{1}^{q}}{\Gamma(q+1)}\left\|h_{1}\right\|_{L^{\infty}}
\end{aligned}
$$


for all $t \in J_{1}$. Taking supremum over $t$, we have

$$
\left\|B_{1} u\right\| \leq\left|\frac{u_{0}}{f\left(0, u_{0}\right)}\right|+\frac{t_{1}^{q}}{\Gamma(q+1)}\left\|h_{1}\right\|_{L^{\infty}}
$$

for all $u \in S_{1}$. We have obtained that $B_{1}\left(S_{1}\right)$ is uniformly bounded.

Let $t_{11}, t_{12} \in J_{1}$ with $t_{11}<t_{12}$. Then, for any $u \in S_{1}$, we have

$$
\begin{aligned}
& \left|B_{1} u\left(t_{11}\right)-B_{1} u\left(t_{12}\right)\right| \\
& \leq\left|\frac{1}{\Gamma(q)} \int_{0}^{t_{11}}\left(t_{11}-s\right)^{q-1} g(s, u(s)) d s-\frac{1}{\Gamma(q)} \int_{0}^{t_{11}}\left(t_{12}-s\right)^{q-1} g(s, u(s)) d s\right| \\
& \quad+\left|\frac{1}{\Gamma(q)} \int_{0}^{t_{11}}\left(t_{12}-s\right)^{q-1} g(s, u(s)) d s-\frac{1}{\Gamma(q)} \int_{0}^{t_{12}}\left(t_{12}-s\right)^{q-1} g(s, u(s)) d s\right| \\
& \leq \frac{\left\|h_{1}\right\|_{L^{\infty}}}{\Gamma(q+1)}\left[\left|t_{12}^{q}-t_{11}^{q}-\left(t_{12}-t_{11}\right)^{q}\right|+\left(t_{12}-t_{11}\right)^{q}\right] .
\end{aligned}
$$

Hence, for $\varepsilon>0$, there exists a constant $\delta>0$ such that

$$
\left|t_{11}-t_{12}\right|<\delta \Rightarrow\left|B_{1} u\left(t_{11}\right)-B_{1} u\left(t_{12}\right)\right|<\varepsilon
$$

for all $t_{11}, t_{12} \in\left[0, t_{1}\right]$ and for all $u \in S_{1}$. This obtains that $B_{1}\left(S_{1}\right)$ is an equicontinuous set in $X_{1}$. By the Arzela-Ascoli theorem, we know that $B_{1}$ is compact. As a result $B_{1}$ is a complete continuous operator on $S_{1}$.

Next, we show that hypothesis (c) of Lemma 2.4 is satisfied. Let $u_{11} \in X_{1}$ and any $u_{12} \in S_{1}$ such that $u_{11}=A_{1} u_{11} B_{1} u_{12}$. Then, by assumption (H2), we have

$$
\begin{aligned}
\left|u_{11}(t)\right| \leq & {\left[\left|f\left(t, u_{11}(t)\right)-f(t, 0)\right|+|f(t, 0)|\right]\left[\left|\frac{u_{0}}{f\left(0, u_{0}\right)}\right|\right.} \\
& \left.+\frac{1}{\Gamma(q)} \int_{0}^{t}(t-s)^{q-1}\left|g\left(s, u_{12}(s)\right)\right| d s\right] \\
\leq & {\left[L_{1}\left|u_{11}(t)\right|+F_{0}\right]\left[\left|\frac{u_{0}}{f\left(0, u_{0}\right)}\right|+\frac{1}{\Gamma(q)} \int_{0}^{t}(t-s)^{q-1} h_{1}(s) d s\right] } \\
\leq & {\left[L_{1}\left|u_{11}(t)\right|+F_{0}\right]\left[\left|\frac{u_{0}}{f\left(0, u_{0}\right)}\right|+\frac{t_{1}^{q}}{\Gamma(q+1)}\left\|h_{1}\right\|_{L^{\infty}}\right] . }
\end{aligned}
$$

Thus,

$$
\left|u_{11}(t)\right| \leq \frac{F_{0}\left(\left|u_{0}\right| \Gamma(q+1)+t_{1}^{q}\left|f\left(0, u_{0}\right)\right|\left\|h_{1}\right\|_{L^{\infty}}\right)}{\Gamma(q+1)\left|f\left(0, u_{0}\right)\right|-L_{1}\left(\left|u_{0}\right| \Gamma(q+1)+t_{1}^{q}\left|f\left(0, t_{0}\right)\right|\left\|h_{1}\right\|_{L^{\infty}}\right)} .
$$

Taking supremum over $t$, we have

$$
\left\|u_{11}\right\| \leq \frac{F_{0}\left(\left|u_{0}\right| \Gamma(q+1)+t_{1}^{q}\left|f\left(0, t_{0}\right)\right|\left\|h_{1}\right\|_{L^{\infty}}\right)}{\Gamma(q+1)\left|f\left(0, u_{0}\right)\right|-L_{1}\left(\left|u_{0}\right| \Gamma(q+1)+t_{1}^{q}\left|f\left(0, t_{0}\right)\right|\left\|h_{1}\right\|_{L^{\infty}}\right)}=N_{1} .
$$

Finally, we have

$$
M_{1}=\left\|B_{1}\left(S_{1}\right)\right\|=\sup \left\{\left\|B_{1} u\right\|: u \in S_{1}\right\} \leq\left|\frac{u_{0}}{f\left(0, u_{0}\right)}\right|+\frac{t_{1}^{q}}{\Gamma(q+1)}\left\|h_{1}\right\|_{L^{\infty}},
$$


and so

$$
L_{1} M_{1} \leq L_{1}\left[\left|\frac{u_{0}}{f\left(0, u_{0}\right)}\right|+\frac{t_{1}^{q}}{\Gamma(q+1)}\left\|h_{1}\right\|_{L^{\infty}}\right]<1 .
$$

So, the operator equation $A_{1} u B_{1} u=u$ has a solution denoted by $u_{1}(t)$ in $S_{1}$.

If $t \in J_{2}$, we set

$$
\begin{aligned}
u(t)= & f(t, u(t))\left[\frac{u_{0}}{f\left(0, u_{0}\right)}+\frac{I_{1}\left(u_{1}\left(t_{1}\right)\right)}{f\left(t_{1}, u_{1}\left(t_{1}\right)\right)}+\frac{1}{\Gamma(q)} \int_{0}^{t_{1}}(t-s)^{q-1} g\left(s, u_{1}(s)\right) d s\right] \\
& +f(t, u(t)) \int_{t_{1}}^{t}(t-s)^{q-1} g(s, u(s)) d s .
\end{aligned}
$$

Set $X_{2}=C\left(J_{2}, \mathbb{R}\right)$ and

$$
S_{2}=\left\{u \in X_{2} \mid\|u\| \leq N_{2}\right\}
$$

where

$$
\begin{aligned}
& N_{2}=\frac{F_{0} H_{2}}{1-L_{2} H_{2}}, \\
& H_{2}=\left|\frac{u_{0}}{f\left(0, u_{0}\right)}+\frac{I_{1}\left(u_{1}\left(t_{1}\right)\right)}{f\left(t_{1}, u_{1}\left(t_{1}\right)\right)}\right|+\frac{t_{2}^{q}}{\Gamma(q+1)}\left\|h_{1}\right\|_{L^{\infty}}+\frac{\left(t_{2}-t_{1}\right)^{q}}{\Gamma(q+1)}\left\|h_{2}\right\|_{L^{\infty}} .
\end{aligned}
$$

Define two operators $A_{2}: X_{2} \mapsto X_{2}$ and $B_{2}: S_{2} \mapsto X_{2}$ by

$$
A_{2} u(t)=f(t, u(t)), \quad t \in J_{2}
$$

and

$$
\begin{aligned}
B_{2} u(t)= & \frac{u_{0}}{f\left(0, u_{0}\right)}+\frac{I_{1}\left(u_{1}\left(t_{1}\right)\right)}{f\left(t_{1}, u_{1}\left(t_{1}\right)\right)}+\frac{1}{\Gamma(q)} \int_{0}^{t_{1}}(t-s)^{q-1} g\left(s, u_{1}(s)\right) d s \\
& +\frac{1}{\Gamma(q)} \int_{t_{1}}^{t}(t-s)^{q-1} g(s, u(s)) d s, \quad t \in J_{2} .
\end{aligned}
$$

The operators $A_{2}$ and $B_{2}$ satisfy all the conditions of Lemma 2.4. First, we prove that $A_{2}$ is a Lipschitz operator on $X_{2}$ with the Lipschitz constant $L_{2}$. Set $u_{21}, u_{22} \in X_{2}$. Then, by hypothesis (H2), we have

$$
\begin{aligned}
\left|A_{2} u_{21}(t)-A_{2} u_{22}(t)\right| & \leq L_{2}\left|u_{21}(t)-u_{22}(t)\right| \\
& \leq L_{2}\left\|u_{21}-u_{22}\right\|
\end{aligned}
$$

for all $t \in J_{2}$. Taking supremum over $t$, we have

$$
\left\|A_{2} u_{21}-A_{2} u_{22}\right\| \leq L_{2}\left\|u_{21}-u_{22}\right\|
$$

for all $u_{21}, u_{22} \in X_{2}$.

Next, we prove that $B_{2}$ is a compact and continuous operator on $S_{2}$ into $X_{2}$. We show that $B_{2}$ is continuous on $S_{2}$. Let $\left\{u_{n}\right\} \subseteq S_{2}$ converge to a point $u \in B_{2}$. Then, by the Lebesgue 
dominated convergence theorem, we have

$$
\begin{aligned}
\lim _{n \rightarrow \infty} B_{2} u_{n}(t)= & \frac{u_{0}}{f\left(0, u_{0}\right)}+\frac{I_{1}\left(u_{1}\left(t_{1}\right)\right)}{f\left(t_{1}, u_{1}\left(t_{1}\right)\right)}+\frac{1}{\Gamma(q)} \int_{0}^{t_{1}}(t-s)^{q-1} g\left(s, u_{1}(s)\right) d s \\
& +\frac{1}{\Gamma(q)} \int_{t_{1}}^{t}(t-s)^{q-1} \lim _{n \rightarrow \infty} g\left(s, u_{n}(s)\right) d s \\
= & \frac{u_{0}}{f\left(0, u_{0}\right)}+\frac{I_{1}\left(u_{1}\left(t_{1}\right)\right)}{f\left(t_{1}, u_{1}\left(t_{1}\right)\right)}+\frac{1}{\Gamma(q)} \int_{0}^{t_{1}}(t-s)^{q-1} g\left(s, u_{1}(s)\right) d s \\
& +\frac{1}{\Gamma(q)} \int_{t_{1}}^{t}(t-s)^{q-1} g(s, u(s)) d s \\
= & B_{2} u(t)
\end{aligned}
$$

for all $t \in J_{2}$. So, $B_{2}$ is continuous on $S_{2}$.

We will prove that $B_{2}$ is a compact operator on $S_{2}$. We show that $B_{2}\left(S_{2}\right)$ is a uniformly bounded and equicontinuous set in $S_{2}$. Let $u \in S_{2}$ be arbitrary. Then, by hypothesis (H3), we have

$$
\begin{aligned}
\left|B_{2} u(t)\right| \leq & \left|\frac{u_{0}}{f\left(0, u_{0}\right)}+\frac{I_{1}\left(u_{1}\left(t_{1}\right)\right)}{f\left(t_{1}, u_{1}\left(t_{1}\right)\right)}\right|+\left|\frac{1}{\Gamma(q)} \int_{0}^{t_{1}}(t-s)^{q-1} g\left(s, u_{1}(s)\right) d s\right| \\
& +\left|\frac{1}{\Gamma(q)} \int_{t_{1}}^{t}(t-s)^{q-1} g(s, u(s)) d s\right| \\
\leq & \left|\frac{u_{0}}{f\left(0, u_{0}\right)}+\frac{I_{1}\left(u_{1}\left(t_{1}\right)\right)}{f\left(t_{1}, u_{1}\left(t_{1}\right)\right)}\right|+\frac{1}{\Gamma(q)} \int_{0}^{t_{1}}(t-s)^{q-1}\left|g\left(s, u_{1}(s)\right)\right| d s \\
& +\frac{1}{\Gamma(q)} \int_{t_{1}}^{t}(t-s)^{q-1}|g(s, u(s))| d s \\
\leq & \left|\frac{u_{0}}{f\left(0, u_{0}\right)}+\frac{I_{1}\left(u_{1}\left(t_{1}\right)\right)}{f\left(t_{1}, u_{1}\left(t_{1}\right)\right)}\right|+\frac{1}{\Gamma(q)} \int_{0}^{t_{1}}(t-s)^{q-1} h_{1}(s) d s \\
& +\frac{1}{\Gamma(q)} \int_{t_{1}}^{t}(t-s)^{q-1} h_{2}(s) d s \\
\leq & \left|\frac{u_{0}}{f\left(0, u_{0}\right)}+\frac{I_{1}\left(u_{1}\left(t_{1}\right)\right)}{f\left(t_{1}, u_{1}\left(t_{1}\right)\right)}\right|+\frac{t_{2}^{q}}{\Gamma(q+1)}\left\|h_{1}\right\|_{L^{\infty}} \\
& +\frac{\left(t_{2}-t_{1}\right)^{q}}{\Gamma(q+1)}\left\|h_{2}\right\|_{L^{\infty}}
\end{aligned}
$$

for all $t \in J_{2}$. Taking supremum over $t$, we have

$$
\left\|B_{2} u\right\| \leq\left|\frac{u_{0}}{f\left(0, u_{0}\right)}+\frac{I_{1}\left(u_{1}\left(t_{1}\right)\right)}{f\left(t_{1}, u_{1}\left(t_{1}\right)\right)}\right|+\frac{t_{2}^{q}}{\Gamma(q+1)}\left\|h_{1}\right\|_{L^{\infty}}+\frac{\left(t_{2}-t_{1}\right)^{q}}{\Gamma(q+1)}\left\|h_{2}\right\|_{L^{\infty}}
$$

for all $u \in S_{2}$. So, $B_{2}\left(S_{2}\right)$ is uniformly bounded.

Let $t_{21}, t_{22} \in J_{2}$ with $t_{21}<t_{22}$. Then, for any $u \in S_{2}$, we have

$$
\begin{aligned}
& \left|B_{2} u\left(t_{21}\right)-B_{2} u\left(t_{22}\right)\right| \\
& \quad \leq\left|\frac{1}{\Gamma(q)} \int_{0}^{t_{1}}\left(t_{21}-s\right)^{q-1} g\left(s, u_{1}(s)\right) d s-\frac{1}{\Gamma(q)} \int_{0}^{t_{1}}\left(t_{22}-s\right)^{q-1} g\left(s, u_{1}(s)\right) d s\right|
\end{aligned}
$$




$$
\begin{aligned}
& +\left|\frac{1}{\Gamma(q)} \int_{t_{1}}^{t_{21}}\left(t_{21}-s\right)^{q-1} g(s, u(s)) d s-\frac{1}{\Gamma(q)} \int_{t_{1}}^{t_{21}}\left(t_{22}-s\right)^{q-1} g(s, u(s)) d s\right| \\
& +\left|\frac{1}{\Gamma(q)} \int_{t_{1}}^{t_{21}}\left(t_{22}-s\right)^{q-1} g(s, u(s)) d s-\frac{1}{\Gamma(q)} \int_{t_{1}}^{t_{22}}\left(t_{22}-s\right)^{q-1} g(s, u(s)) d s\right| \\
\leq & \frac{\left\|h_{1}\right\|_{L^{\infty}}}{\Gamma(q+1)}\left|t_{22}^{q}-t_{21}^{q}+\left(t_{22}-t_{1}\right)^{q}-\left(t_{21}-t_{1}\right)^{q}\right| \\
& +\frac{\left\|h_{2}\right\|_{L^{\infty}}}{\Gamma(q+1)}\left[\left|\left(t_{22}-t_{1}\right)^{q}-\left(t_{21}-t_{1}\right)^{q}+\left(t_{22}-t_{21}\right)^{q}\right|+\left(t_{22}-t_{21}\right)^{q}\right] .
\end{aligned}
$$

Hence, for $\varepsilon>0$, there exists a constant $\delta>0$ such that

$$
\left|t_{21}-t_{22}\right|<\delta \Rightarrow\left|B_{2} u\left(t_{21}\right)-B_{2} u\left(t_{22}\right)\right|<\varepsilon
$$

for all $t_{21}, t_{22} \in J_{2}$ and for all $u \in S_{2}$. So, $B_{2}\left(S_{2}\right)$ is an equicontinuous set in $X_{2}$. By the ArzelaAscoli theorem, we know that $B_{2}$ is compact. As a result, $B_{2}$ is a complete continuous operator on $S_{2}$.

Next, we show that hypothesis (c) of Lemma 2.4 is satisfied. Let $u_{21} \in X_{2}$ and any $u_{22} \in S_{2}$ such that $u_{21}=A_{2} u_{21} B_{2} u_{22}$. Then, by assumptions (H2) and (H3), we have

$$
\begin{aligned}
\left|u_{21}(t)\right| \leq & {\left[\left|f\left(t, u_{21}(t)\right)-f(t, 0)\right|+|f(t, 0)|\right]\left[\left|\frac{u_{0}}{f\left(0, u_{0}\right)}+\frac{I_{1}\left(u_{1}\left(t_{1}\right)\right)}{f\left(t_{1}, u_{1}\left(t_{1}\right)\right)}\right|\right.} \\
& \left.+\frac{1}{\Gamma(q)} \int_{0}^{t_{1}}(t-s)^{q-1}\left|g\left(s, u_{1}(s)\right)\right| d s+\frac{1}{\Gamma(q)} \int_{t_{1}}^{t}(t-s)^{q-1}\left|g\left(s, u_{22}(s)\right)\right| d s\right] \\
\leq & {\left[L_{2}\left|u_{21}(t)\right|+F_{0}\right]\left[\left|\frac{u_{0}}{f\left(0, u_{0}\right)}+\frac{I_{1}\left(u_{1}\left(t_{1}\right)\right)}{f\left(t_{1}, u_{1}\left(t_{1}\right)\right)}\right|\right.} \\
& \left.+\frac{1}{\Gamma(q)} \int_{0}^{t_{1}}(t-s)^{q-1} h_{1}(s) d s+\frac{1}{\Gamma(q)} \int_{t_{1}}^{t}(t-s)^{q-1} h_{2}(s) d s\right] \\
\leq & {\left[L_{2}\left|u_{21}(t)\right|+F_{0}\right]\left[\left|\frac{u_{0}}{f\left(0, u_{0}\right)}+\frac{I_{1}\left(u_{1}\left(t_{1}\right)\right)}{f\left(t_{1}, u_{1}\left(t_{1}\right)\right)}\right|\right.} \\
& \left.+\frac{t_{2}^{q}}{\Gamma(q+1)}\left\|h_{1}\right\|_{L^{\infty}}+\frac{\left(t_{2}-t_{1}\right)^{q}}{\Gamma(q+1)}\left\|h_{2}\right\|_{L^{\infty}}\right] .
\end{aligned}
$$

Thus,

$$
\left|u_{21}(t)\right| \leq \frac{F_{0} H_{2}}{1-L_{2} H_{2}}
$$

Taking supremum over $t$, we have

$$
\left\|u_{21}\right\| \leq \frac{F_{0} H_{2}}{1-L_{2} H_{2}}=N_{2}
$$

Finally, we have

$$
M_{2} \leq\left|\frac{u_{0}}{f\left(0, u_{0}\right)}+\frac{I_{1}\left(u_{1}\left(t_{1}\right)\right)}{f\left(t_{1}, u_{1}\left(t_{1}\right)\right)}\right|+\frac{t_{2}^{q}}{\Gamma(q+1)}\left\|h_{1}\right\|_{L^{\infty}}+\frac{\left(t_{2}-t_{1}\right)^{q}}{\Gamma(q+1)}\left\|h_{2}\right\|_{L^{\infty}}
$$


and

$$
\begin{aligned}
L_{2} M_{2} \leq & L_{2}\left[\left|\frac{u_{0}}{f\left(0, u_{0}\right)}+\frac{I_{1}\left(u_{1}\left(t_{1}\right)\right)}{f\left(t_{1}, u_{1}\left(t_{1}\right)\right)}\right|+\frac{t_{2}^{q}}{\Gamma(q+1)}\left\|h_{1}\right\|_{L^{\infty}}\right. \\
& \left.+\frac{\left(t_{2}-t_{1}\right)^{q}}{\Gamma(q+1)}\left\|h_{2}\right\|_{L^{\infty}}\right]<1 .
\end{aligned}
$$

So, the operator equation $A_{2} u B_{2} u=u$ has a solution denoted by $u_{2}(t)$ in $S_{2}$.

If $t \in j_{k+1}(k=2,3, \ldots, m)$, repeating the same process, we obtain $u_{(k+1)}(t) \in C\left(J_{k+1}, \mathbb{R}\right)$ $(k=2,3, \ldots, m)$. So, we get a mild solution of IHFDE (4). The proof is completed.

\section{Example}

In this section we give a simple example to illustrate the usefulness of our main result.

Example 4.1 Let us consider the impulsive hybrid fractional differential equations

$$
\left\{\begin{array}{l}
{ }^{c} D_{0, t}^{q}\left[\frac{u(t)}{e^{v_{1} t}+|u(t)|}\right]=\frac{e^{-v_{2} t}|u(t)|}{\left(1+e^{t}\right)(1+|u(t)|)}, \quad t \in[0,1] \backslash t_{1}, \\
u\left(t_{1}^{+}\right)=u\left(t_{1}^{-}\right)+\left(-2 u\left(t_{1}^{-}\right)\right), \quad t_{1} \neq 0,1 \\
u(0)=0,
\end{array}\right.
$$

where $v_{1}>0, v_{2}>0$ are constants and $0<q<1$.

Set

$$
f(t, u(t))=e^{v_{1} t}+|u(t)|, \quad g(t, u(t))=\frac{e^{-v_{2} t}|u(t)|}{\left(1+e^{t}\right)(1+|u(t)|)}
$$

for all $(t, u) \in[0,1] \times \mathbb{R}$.

Obviously,

$$
f(t, u(t)) \neq 0 \text { and } f\left(t_{1}^{+}, u\left(t_{1}^{+}\right)\right)=f\left(t_{1}^{-}, u\left(t_{1}^{-}\right)\right)
$$

for all $(t, u) \in[0,1] \times \mathbb{R}$. There exist constants $L_{k}=1(k=1,2)$ such that

$$
\left|f\left(t, u_{1}\right)-f\left(t, u_{2}\right)\right| \leq L_{k}\left|u_{1}-u_{2}\right|
$$

for all $t \in J_{k}(k=1,2)$ and $u_{1}, u_{2} \in \mathbb{R}$.

By

$$
\frac{u(t)}{f(t, u(t))}= \begin{cases}1-\frac{e^{v_{1} t}}{e^{v_{1} t}+u(t)}, & u(t) \geq 0, \\ -1+\frac{e^{v_{1}}}{e^{v_{1} t}-u(t)}, & u(t)<0,\end{cases}
$$

we have that the function $u \mapsto \frac{u}{f(t, u(t))}$ is increasing in $\mathbb{R}$ for $\left[0, t_{1}\right]$.

For all $u \in \mathbb{R}$ and each $t \in[0,1]$, we have

$$
g(t, u(t))=\frac{e^{-v t}|u(t)|}{\left(1+e^{t}\right)(1+|u(t)|)} \leq \frac{e^{-v t}}{1+e^{t}} \leq \frac{e^{-v t}}{2} .
$$

So, choosing some $v_{1}, v_{2}>0$ large enough, we have

$$
L_{2}\left[\left|\frac{-2 u\left(t_{1}^{-}\right)}{e^{v_{1} t_{1}}+\left|u\left(t_{1}\right)\right|}\right|+\frac{1}{\Gamma(q+1)}\left(\left(1-t_{1}\right)^{q}\left\|h_{1}\right\|_{L^{1}}+1\right)\left\|h_{2}\right\|_{L^{1}}\right]<1 \text {. }
$$


Thus all the assumptions in Theorem 3.1 are satisfied, our results can be applied to problem (45).

\section{Competing interests}

The authors declare that they have no competing interests.

\section{Authors' contributions}

The authors declare that the study was realized in collaboration with the same responsibility. All authors read and approved the final manuscript.

\section{Acknowledgements}

The authors thank the referees for their careful reading of the manuscript and insightful comments, which helped to improve the quality of the paper. We would also like to acknowledge the valuable comments and suggestions from the editors, which vastly contributed to improving the presentation of the paper. This work was supported by the NSF of China (Nos. 11371183, 11271050) and the NSF of Shandong Province (No. ZR2013AM004).

Received: 10 March 2014 Accepted: 11 July 2014 Published: 04 Aug 2014

\section{References}

1. Diethelm, K: The Analysis of Fractional Differential Equations. Lecture Notes in Mathematics (2010)

2. Kilbas, AA, Srivastava, HM, Trujillo, JJ: Theory and Applications of Fractional Differential Equations. Elsevier, Amsterdam (2006)

3. Lakshmikantham, V, Leela, S, Vasundhara Devi, J: Theory of Fractional Dynamic Systems. Cambridge Scientific Publishers, Cambridge (2009)

4. Miller, KS, Ross, B: An Introduction to the Fractional Calculus and Differential Equations. Wiley, New York (1993)

5. Michalski, MW: Derivatives of noninteger order and their applications. Diss. Math. 328, 1-47 (1993)

6. Podlubny, I: Fractional Differential Equations. Academic Press, San Diego (1999)

7. Tarasov, VE: Fractional Dynamics: Application of Fractional Calculus to Dynamics of Particles, Fields and Media. Springer, Berlin (2011)

8. Agarwal, RP, Benchohra, M, Hamani, S: A survey on existence results for boundary value problems of nonlinear fractional differential equations and inclusions. Acta Appl. Math. 109, 973-1033 (2010)

9. Ahmad, B, Nieto, JJ: Existence of solutions for anti-periodic boundary value problems involving fractional differential equations via Leray-Schauder degree theory. Topol. Methods Nonlinear Anal. 35, 295-304 (2010)

10. Bai, Z: On positive solutions of a nonlocal fractional boundary value problem. Nonlinear Anal. TMA 72, 916-924 (2010)

11. Benchohra, M, Henderson, J, Ntouyas, SK, Ouahab, A: Existence results for fractional order functional differential equations with infinite delay. J. Math. Anal. Appl. 338, 1340-1350 (2008)

12. Mophou, GM, N'Guérékata, GM: Existence of mild solutions of some semilinear neutral fractional functional evolution equations with infinite delay. Appl. Math. Comput. 216, 61-69 (2010)

13. Wang, J, Zhou, Y: A class of fractional evolution equations and optimal controls. Nonlinear Anal., Real World Appl. 12 , 262-272 (2011)

14. Wang, J, Zhou, Y, Wei, W: A class of fractional delay nonlinear integrodifferential controlled systems in Banach spaces. Commun. Nonlinear Sci. Numer. Simul. 16, 4049-4059 (2011)

15. Zhang, S: Existence of positive solution for some class of nonlinear fractional differential equations. J. Math. Anal. Appl. 278, 136-148 (2003)

16. Zhou, Y, Jiao, F: Nonlocal Cauchy problem for fractional evolution equations. Nonlinear Anal., Real World Appl. 11 4465-4475 (2010)

17. Zhou, Y, Jiao, F, Li, J: Existence and uniqueness for $p$-type fractional neutral differential equations. Nonlinear Anal. TMA 71, 2724-2733 (2009)

18. Zhou, Y, Jiao, F, Li, J: Existence and uniqueness for fractional neutral differential equations with infinite delay. Nonlinear Anal. TMA 71, 3249-3256 (2009)

19. Dhage, BC: On $\alpha$-condensing mappings in Banach algebras. Math. Stud. 63, 146-152 (1994)

20. Dhage, BC, Lakshmikantham, V: Basic results on hybrid differential equations. Nonlinear Anal. Hybrid Syst. 4, 414-424 (2010)

21. Dhage, BC: A nonlinear alternative in Banach algebras with applications to functional differential equations. Nonlinear Funct. Anal. Appl. 8, 563-575 (2004)

22. Dhage, BC: Fixed point theorems in ordered Banach algebras and applications. Panam. Math. J. 9, $93-102$ (1999)

23. Zhao, Y, Sun, S, Han, Z, Li, Q: Theory of fractional hybrid differential equations. Comput. Math. Appl. 62, 1312-1324 (2011)

24. Fečkan, M, Zhou, Y, Wang, J: On the concept and existence of solutions for impulsive fractional differential equations. Commun. Nonlinear Sci. Numer. Simul. 17, 3050-3060 (2012)

25. Herzallah, MAE, Baleanu, D: On fractional order hybrid differential equations. Abstr. Appl. Anal. 2014, Article ID 389386 (2014)

26. Fečkan, M, Zhou, Y, Wang, J: Response to 'Comments on the concept of existence of solution for impulsive fractional differential equations [Commun Nonlinear Sci Numer Simul 2014; 19:401-3.]'. Commun. Nonlinear Sci. Numer. Simul. $19,4213-4215(2014)$

27. Wang, J, Ibrahim, AG, Fečkan, M: Nonlocal impulsive fractional differential inclusions with fractional sectorial operators on Banach spaces. Appl. Math. Comput. (2014). doi:10.1016/j.amc.2014.04.093

28. Dhage, BC: On a fixed point theorem in Banach algebras with applications. Appl. Math. Lett. 18, 273-280 (2005) 
10.1186/1687-1847-2014-211

Cite this article as: Ge and Xin: On the existence of a mild solution for impulsive hybrid fractional differential equations. Advances in Difference Equations 2014, 2014:211

Submit your manuscript to a SpringerOpen ${ }^{\circ}$ journal and benefit from:

- Convenient online submission

- Rigorous peer review

- Immediate publication on acceptance

Open access: articles freely available online

- High visibility within the field

- Retaining the copyright to your article 